\title{
Explorando el océano a través de paisajes sonoros
}

\author{
Jennifer L. Miksis-Olds ${ }^{A}$, Bruce Martin ${ }^{B}$, Peter L. Tyack ${ }^{C}$ \\ A School of Marine Science and Ocean Engineering University of New \\ Hampshire, Estados Unidos de América \\ B JASCO Applied Sciences, Canadá \\ C Sea Mammal Research Unit Scottish Oceans Institute School of Biology \\ University of St Andrews Fife St Andrews, Escocia, Reino Unido \\ j.miksisolds@unh.edu; bruce.martin@jasco.com; plt@st-andrews.ac.uk
}

\section{RESUMEN}

Escuchar paisajes sonoros submarinos nos ayuda a entender cómo la fisica oceánica y la biología de las comunidades marinas están respondiendo a un océano que cambia dinámicamente.

\section{PALABRAS CLAVE}

Paisajes sonoros, señales acústicas en los océanos.

\begin{abstract}
Listening to underwater soundscapes helps us understand how ocean physics and the biology of marine communities are responding to a dynamically changing ocean.
\end{abstract}

\section{KEYWORDS}

Ocean soundscapes, acoustic signals in the oceans.

\section{INTRODUCCIÓN}

Es una tarde clara y usted está mirando hacia el horizonte desde el punto más alto dentro de $100 \mathrm{~km}$. Se pueden ver "millas y millas", pero los únicos sonidos que se pueden escuchar son la gente con usted, algunos pájaros, insectos y el viento. Ahora, si usted fuera a un punto equivalente en el océano para estar parado en la cresta del medio Atlántico con vista a la llanura abisal del océano, usted todavía tendría 1,200 m de agua obscura alrededor. Escuchando a través de un hidrófono, los sonidos serían extraordinariamente ricos. Se oirían los crustáceos arañando la roca y los corales de aguas profundas. Las ballenas de esperma, las ballenas con pico y las ballenas piloto estarían buscando comida mediante ecolocalización. Los llamados de la ballena azul y de aleta, atrapados en el canal de sonido profundo, llegarían de miles de kilómetros de distancia. Cada pocos segundos, el canal de sonido también traería pulsos de energía de los estudios sísmicos de petróleo y gas que llegan desde Brasil, África, el Mar del Norte y Terranova.

La investigación acústica subacuática ha revelado la increíble física de cómo se propaga el sonido en el océano, motivado principalmente por el uso del sonido para detectar petróleo y gas bajo la corteza terrestre o para aplicaciones navales. A lo largo del camino, aprendimos que la vida marina ha aprovechado la física 
oceánica y ha evolucionado en el uso del sonido como una modalidad sensorial primaria para interactuar con el medio ambiente. Ahora estamos escuchando las conversaciones submarinas y utilizando la acústica pasiva para evaluar la biodiversidad marina, la densidad animal y el estado y la salud del ecosistema. Este artículo presenta la idea de un paisaje sonoro submarino, el uso exitoso del paisaje sonoro para entender la ecología marina, el modelado de paisajes sonoros y el sonido oceánico como una variable oceánica esencial (EOV).

\section{PAISAJES SONOROS SUBACUÁTICOS}

Una gran cantidad de información relacionada con la dinámica de los océanos y las actividades humanas puede obtenerse simplemente escuchando el campo de sonido ambiental. Este paisaje acústico, o paisaje sonoro, es la suma de múltiples fuentes sonoras que llegan a la ubicación de un animal receptor o un grabador acústico. Los sonidos medidos en una grabadora acústica se caracterizan por nuestras mediciones típicas de ingeniería tales como niveles de presión acústica, niveles de exposición ponderados del sonido (SELs), rugosidad, y curtosis. La percepción de los sonidos a la vida marina depende de la contribución relativa de cada fuente, dirección de la fuente, propagación a través del entorno, texto conductual, capacidad auditiva del oyente, e historia del oyente con sonidos similares (figura 1).

Fig. 1. Arriba: el paisaje sonoro está compuesto por el sonido, las mediciones físicas del campo sonoro, y el paisaje que transmite cómo todas las fuentes sonoras se superponen y son percibidas por el oyente. Cajas blancas: señales $S_{i(1-7)}$ procedentes de fuentes sonoras en el entorno con diferentes tamaños y orientaciones destinadas a transmitir diferentes tipos de fuentes; cajas negras: características físicas y perceptivas de las señales sonoras del oyente. $\mathrm{L}_{\text {aeq }}$ : nivel sonoro en decibelios equivalente a la energía acústica total ponderada durante un tiempo determinado. ${ }^{1}$ Abajo: representación de las múltiples fuentes oceánicas que forman un paisaje sonoro oceánico. ${ }^{2}$

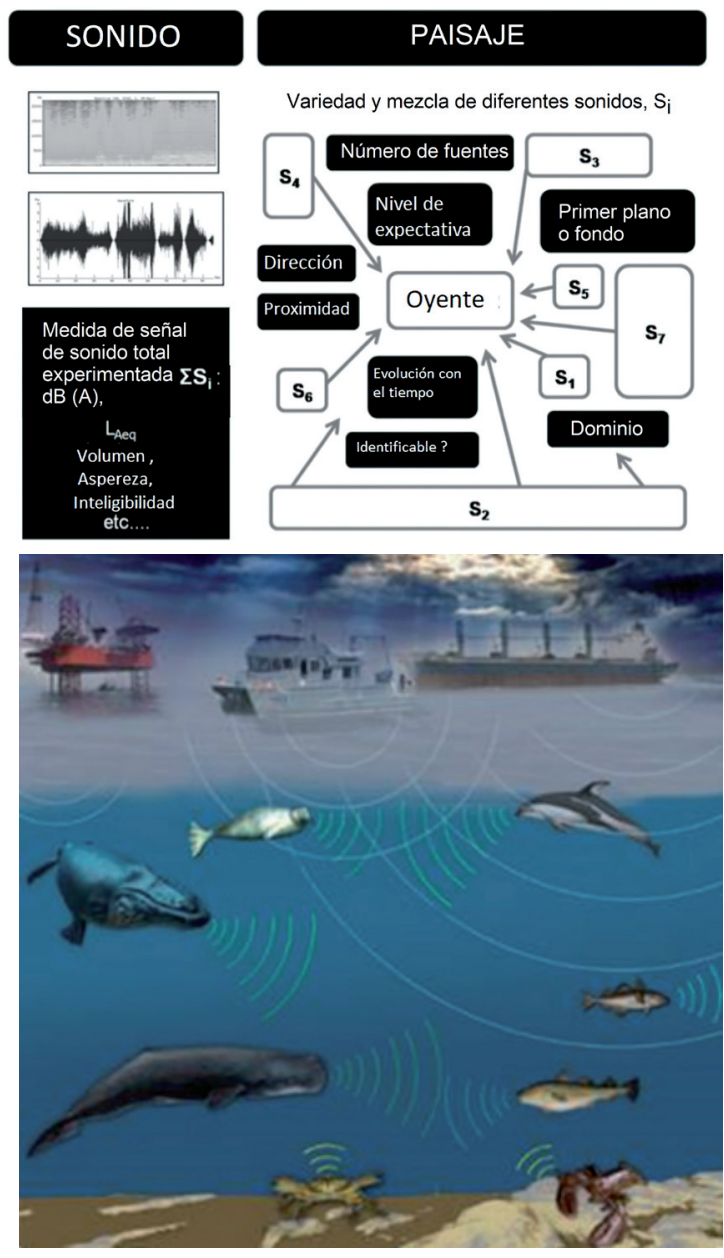


Los paisajes sonoros submarinos son dinámicos; varían en espacio y tiempo y dentro de los hábitats y entre ellos. El sonido en las profundidades del océano se propaga a tales distancias bajo el agua que los paisajes sonoros son influenciados no sólo por las condiciones locales sino también por fuentes sonoras mucho más distantes que en el aire. El paisaje sonoro submarino se compone de contribuciones (figura 2) de la actividad humana (por ejemplo, transporte marítimo, buques pesqueros, muestreadores para estudios sísmicos), procesos naturales abióticos o geofísicos (por ejemplo, viento, lluvia, hielo), y contribuciones acústicas de fuentes biológicas (por ejemplo, sonido producido a partir de movimientos de animales y vocalizaciones de mamíferos marinos, peces e invertebrados). Las flechas en una sola dirección en la figura 2 muestran que el paisaje sonoro está directamente influenciado en una sola dirección por factores antropogénicos y abióticos, y las flechas en dos direcciones indican que el sonido no está sólo influenciado, sino que también influye, sobre el componente biológico del paisaje sonoro. Por consiguiente, el paisaje sonoro subacuático no es simplemente un parámetro físico del medio ambiente que debe medirse y cuantificarse. El paisaje sonoro depende del oyente y tiene un laso de retroalimentación donde los cambios en el paisaje sonoro tienen el potencial de impacto de comportamiento acústico y factores bióticos que influyen en la ecología conductual del ecosistema y finalmente alteran aún más el paisaje sonoro (figura 2).

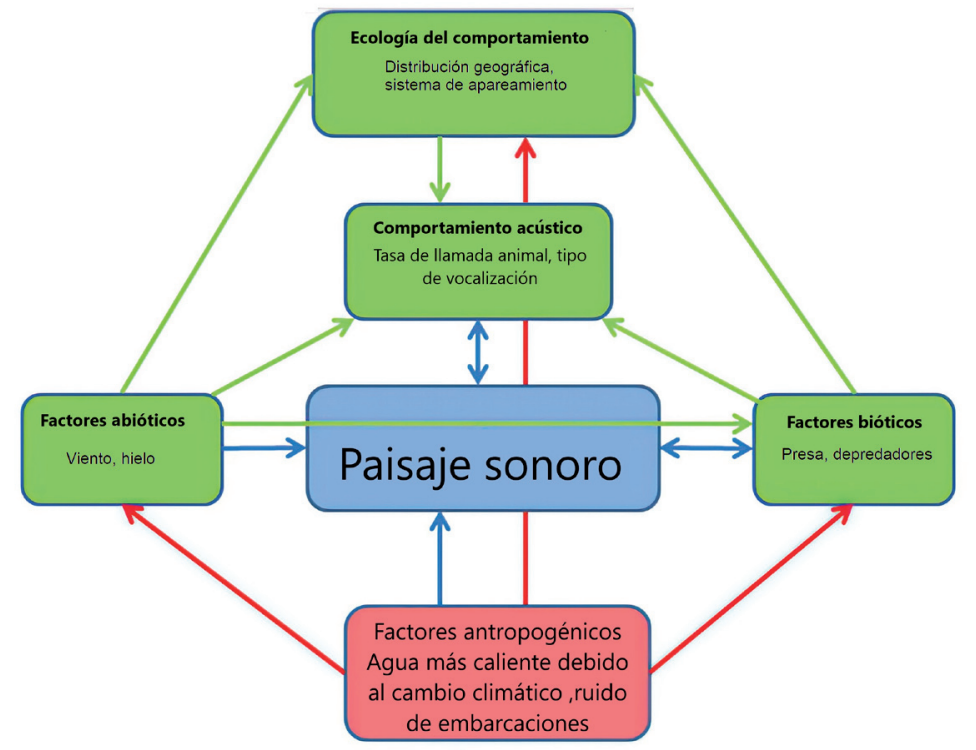

Fig. 2. Paisaje sonoro presentado en el contexto de la ecología acústica. Cajas verdes y flechas, factores naturales: ecología conductual, comportamiento acústico, y factores abióticos y bióticos que contribuyen a (flechas salientes) o impactados por (flechas entrantes) el paisaje sonoro (caja azul y flechas); caja roja y flechas, interacciones y las variaciones de la actividad humana relacionadas con el paisaje sonoro o que repercuten en él. Adaptado de van Opzeeland y Miksis-Olds. ${ }^{3}$

El análisis del paisaje sonoro se realiza en corrientes de datos en directo o grabaciones de señales de presión recibidas de registradores acústicos pasivos desplegados en el fondo del océano o anclados en la columna de agua. Las grabaciones nos permiten observar hábitats marinos sin los efectos desconcertantes 
de la presencia humana o el sesgo del muestreo. Las grabaciones de la gama completa de sonidos oceánicos tienen un ancho de banda amplio (150kHz o más), duran períodos de meses a años, y a menudo se recogen en lugares múltiples que los investigadores comparan para encontrar similitudes y diferencias (escuche paisajes sonoros en tiempo real grabados en diferentes lugares oceánicos en http://www.listentoteep.com). Estos conjuntos de datos son llamados de cinco dimensiones porque tienen tiempo, frecuencia, amplitud, latitud y longitud. El objetivo del análisis del paisaje sonoro es extraer información de las grabaciones para identificar qué fuentes están presentes, las amplitudes de las fuentes, cómo interactúan y cómo los animales en el medio ambiente pueden percibir y responder a los sonidos. En los últimos años, algunos equipos de investigación han comenzado a realizar grabaciones de paisajes sonoros direccionales que aumentan los datos a seis dimensiones añadiendo la dirección de llegada (figura $3)$. Los sensores de presión direccionales en aguas profundas también ofrecen el potencial para medir el movimiento de partículas. Por desgracia, esta metodología no se extiende a mediciones precisas de las partículas cerca de la superficie del mar, en el fondo marino o en aguas poco profundas, ya que no está relacionado linealmente con la presión en estas regiones. El movimiento de partículas, a diferencia de la presión, es el componente del sonido percibido por la mayoría de los peces e invertebrados marinos. Su medición y percepción es un tema que requiere una amplia investigación. ${ }^{4}$

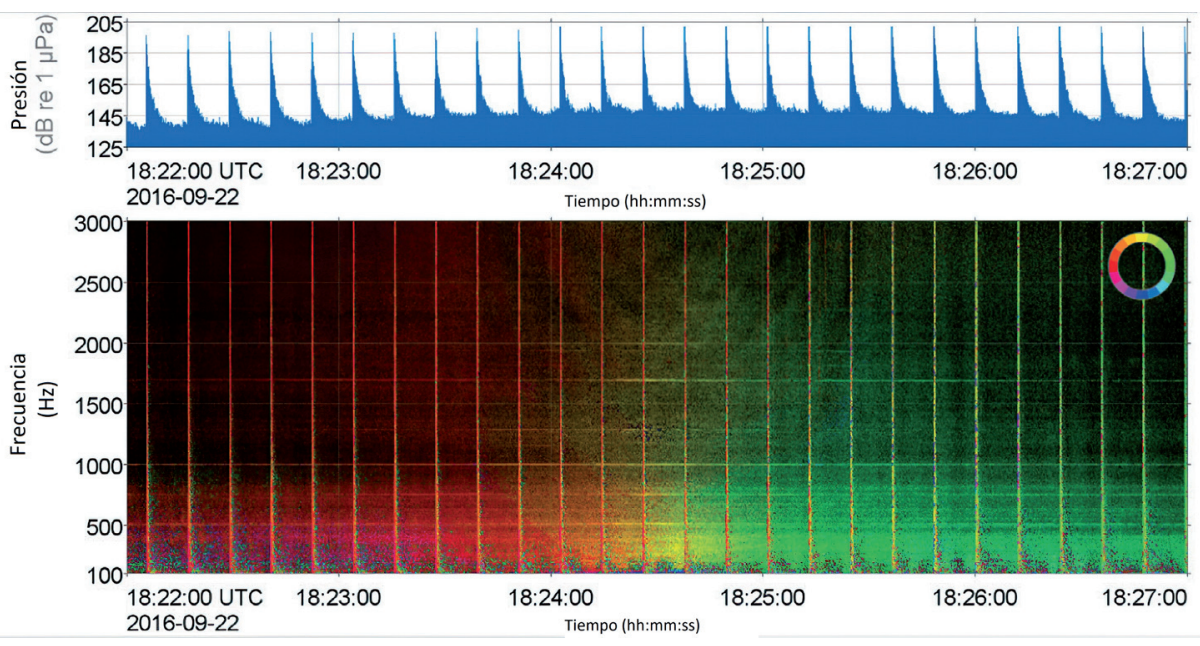

Fig. 3. Cinco minutos de datos acústicos de una nave sísmica y un cañón de aire pasando sobre un hidrófono direccional. Arriba: serie temporal de nivel de presión acústica. Abajo: espectrograma donde el color indica la dirección de llegada mostrada por la rueda de color (amarillo, Norte; azul, Sur). El sensor estaba a $60 \mathrm{~cm}$ del fondo marino en 110 $\mathrm{m}$ de agua. Tenga en cuenta que la dirección del buque cambia de color antes de los impulsos del arreglo del cañón de aire porque estaba $\sim 100 \mathrm{~m}$ detrás de la nave.

Los datos de monitorización acústica pasiva (soundscape) pueden descomponerse de forma selectiva para comprender mejor las fuentes que configuran las características temporales, espaciales y espectrales del entorno acústico ${ }^{5,6}$ (por ejemplo la figura 4). Hay una amplia variedad de medidas acústicas $\mathrm{y}$ formatos de presentación en la literatura de paisajes marinos relacionados con los focos de cada estudio. Por ejemplo, los estudios de patrones y tendencias del 


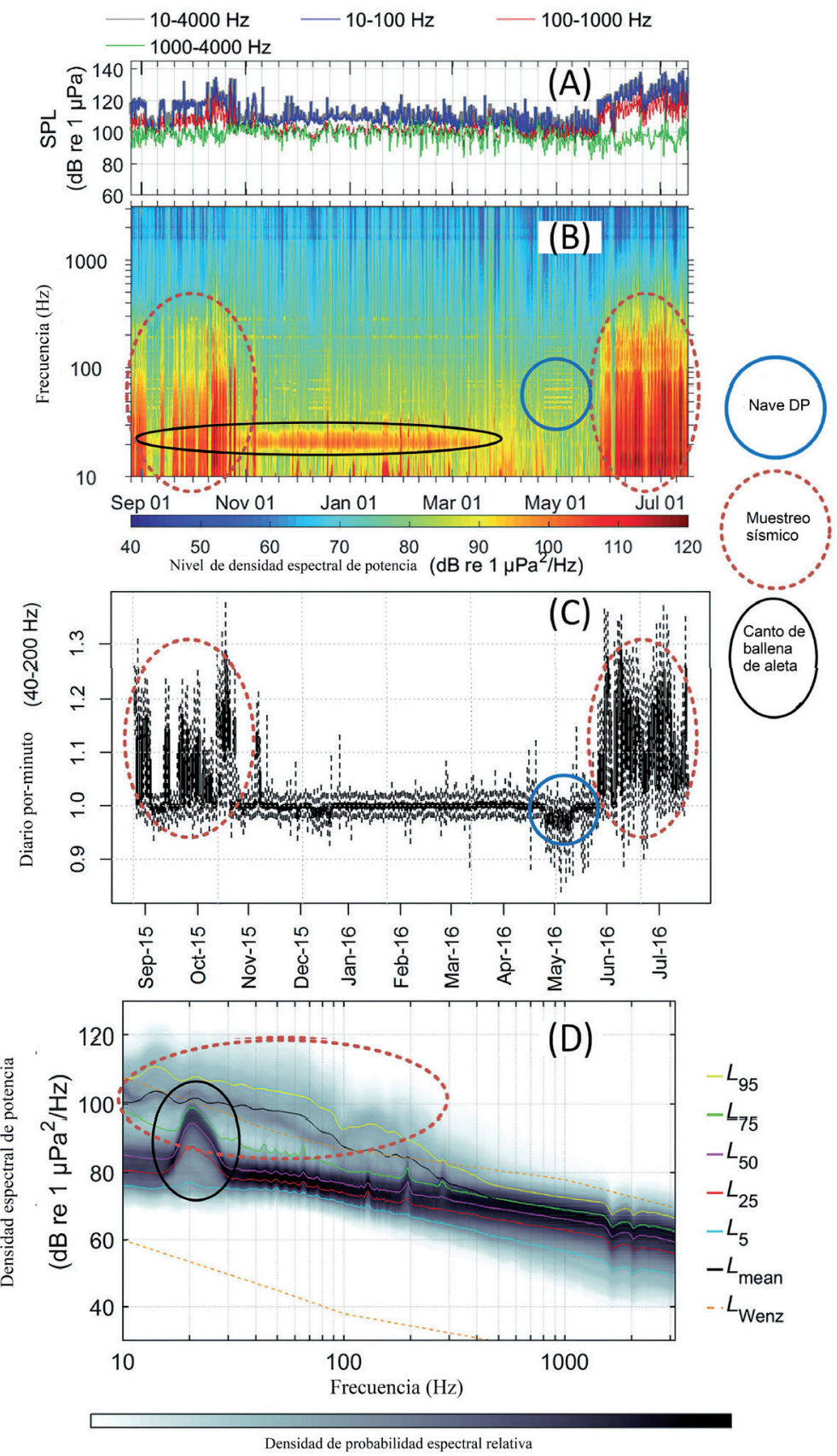

Fig. 4. Ejemplos de presentaciones de datos de paisajes sonoros utilizando un conjunto de datos de 11 meses registrados a $20 \mathrm{~m}$ del lecho marino en 1,280 m de agua frente a Terranova, Canadá. A: serie temporal de niveles de presión acústica limitados en banda de 1 hora (SPL). B: media espectral a largo plazo del conjunto de datos completo. $C$ : distribuciones diarias de valores de índice de complejidad acústica de 1 minuto $(\mathrm{ACl})$ para la banda de frecuencia de $40-200 \mathrm{~Hz}$. Los valores inferiores a 1 indican una menor complejidad (es decir, fuentes sonoras continuas) y los superiores a 1 indican una mayor complejidad (fuentes sonoras impulsivas). D: distribución de densidades espectrales de 1 minuto de potencia, incluidos los percentiles de superación (es decir, el $5 \%$ de los valores de potencia de la densidad espectral superaron la línea L5). Elipses rayadas anaranjadas, presencia de señales sísmicas; elipses sólidas negras, ballenas aletas; círculos azules sólidos, una nave de posicionamiento dinámico distante (DP). 
paisaje sonoro tienden a utilizar mediciones de los niveles de presión acústica (figura 4, A y B) y percentiles de superación del nivel sonoro (nivel sonoro que se supera N\% del tiempo durante un período de tiempo especificado; figura 4D), mientras que los estudios de la biodiversidad de los ecosistemas muestran índices de diversidad acústica del paisaje sonoro representativo del número de especies vocales presentes en una zona (figura 4C). Los análisis de paisajes sonoros han proporcionado un medio para comprender mejor la influencia de los parámetros ambientales como la presencia de hielo marino y los ciclos lunares en los procesos acústicos locales ${ }^{7,8}$ evaluando la calidad del habitat y la salud de los arrecifes de coral ${ }^{8,9}$ y midiendo la biodiversidad ${ }^{10,11}$ para comprender mejor los impactos y riesgos de las contribuciones humanas al paisaje sonoro en la vida marina.

\section{UTILIZACIÓN DE PAISAJES SONOROS SUBMARINOS}

Durante la última década, los costes de recogida y análisis de datos de vigilancia acústica pasiva han disminuido constantemente, lo que ha dado lugar a un número creciente de estudios que exploran cómo los animales utilizan la información de su paisaje sonoro ambiental para la comunicación, orientación y navegación ${ }^{12,13}$ (véase también el artículo de Slabekoorn ${ }^{14}$ en Acoustics Today). El concepto de utilizar sonidos ambientales o reflejados (a diferencia de las señales de comunicación específicas) como señales de movimiento directo o de identificación de hábitats apropiados se ha identificado recientemente como un nuevo campo de estudio denominado orientación del paisaje sonoro, y el concepto también se incluye en el campo más amplio de la ecología del paisaje sonoro en la literatura científica. ${ }^{12,13}$ Se ha especulado que las grandes ballenas barbadas usan señales acústicas ambientales o señales acústicas para guiar su migración. ${ }^{15,16}$ Del mismo modo, se ha propuesto que las señales de los paisajes sonoros puedan proporcionar a las focas en el agua un gradiente acústico destacado entre el agua abierta y las condiciones de hielo sólido mediante el cual las focas pueden orientarse para mantener el acceso al agua abierta para respirar. ${ }^{17}$

Los estudios de laboratorio y sobre el terreno han demostrado que tanto los invertebrados como los peces utilizan señales de los paisajes sonoros para la orientación y localización del hábitat de asentamiento apropiado. Stanley et al. ${ }^{18}$ midieron el nivel de intensidad acústica requerido para provocar asentamiento y metamorfosis en varias especies de cangrejos, y Simpson et al. ${ }^{19}$ mostraron que los peces de arrecifes de coral parecen responder más fuertemente a los componentes de mayor frecuencia $(>570 \mathrm{~Hz})$ del paisaje sonoro del arrecife. Los hábitats con mayor biodiversidad suelen asociarse a paisajes acústicos más ricos en comparación con los hábitats de baja diversidad, que en sí mismos pueden ser una señal importante para la orientación animal en el agua y el aire. ${ }^{13,20,21}$

Un ejemplo de la utilidad del análisis a largo plazo del paisaje sonoro es el estudio de los últimos 50 años en la costa oeste de los Estados Unidos. Tras una combinación de grabaciones desclasificadas de la Marina de los Estados Unidos y conjuntos de datos científicos, se ha documentado un aumento constante del sonido de baja frecuencia $(10-200 \mathrm{~Hz})$ que se atribuye principalmente a un aumento del transporte marítimo comercial. ${ }^{22}$. Los niveles de ruido han aumentado en aproximadamente $3 \mathrm{~dB} /$ década $(0.55 \mathrm{~dB} /$ año $)$ hasta la década de $1980{ }^{23}$ y luego se han reducido a $0.2 \mathrm{~dB} /$ año. ${ }^{24}$ Las mediciones más recientes 
en esta región muestran una nivelación o una ligera disminución de los niveles sonoros desde finales de la década de 1990, a pesar del aumento del número y tamaño de los buques. ${ }^{25}$

La ballena azul, de aleta, jorobada, el rorcual del norte y el Brydes, y el cachalotes se comunican en la banda de frecuencia de 10 a $200 \mathrm{~Hz}$; el infrasonido de las olas estrellándose en tierra (que los animales marinos probablemente utilizan para la orientación) también está en esta banda. La comprensión de cómo la vida marina utiliza esta banda de frecuencia y los efectos de las contribuciones humanas en esta misma banda es el tema de muchos estudios de paisajes sonoros. Los aumentos del transporte marítimo por sí solos no explican plenamente el aumento observado de 10 a $12 \mathrm{~dB}$ en la banda de 20 a $40 \mathrm{~Hz}$ entre 1965 y $2003 .{ }^{22,26}$ Las actividades de exploración y producción de petróleo y gas, así como de fuentes de energía renovables, también han aumentado los niveles sonoros totales en esta banda. ${ }^{27}$ Es probable que los niveles sonoros bióticos también hayan aumentado debido a la recuperación de las poblaciones de ballenas y al efecto Lombard, que es el aumento de la amplitud de las llamadas para compensar los niveles de ruido más elevados. El efecto Lombard se ha demostrado en humanos y en muchas poblaciones animales y puede contribuir a elevar los niveles de baja frecuencia a medida que los animales vocalizan más fuerte para ser escuchados por encima del ruido. ${ }^{28}$

El cambio climático está aumentando la cantidad de hielo glacial que envuelve los océanos, y a medida que los glaciares se desintegran, generan ruido de baja frecuencia con grandes niveles de origen que contribuyen al presupuesto regional de ruido durante largos períodos. ${ }^{29}$ Los límites regionales de los paisajes sonoros, incluso para las frecuencias bajas que propagan grandes distancias, están infravalorados por las diferencias en el aumento del nivel sonoro a largo plazo. Aunque los estudios reportan un aumento significativo de los niveles de ruido ambiental en el Pacífico Norte, los estudios actuales en los océanos Índico, Atlántico Sur y Pacífico ecuatorial no han observado un aumento uniforme del nivel sonoro de los océanos. ${ }^{30,31}$ Se sabe muy poco sobre el paisaje sonoro mundial en su conjunto, y esta es una zona activa de exploración oceánica. La teoría y las observaciones sugieren que el ruido generado por el hombre podría estar acercándose a niveles en los que podría haber efectos negativos sobre la vida marina. ${ }^{27}$

\section{MODELADO DEL PAISAJE SONORO}

Hasta ahora hemos discutido la descomposición de los sonidos medidos en los puntos del océano para explorar qué fuentes están presentes y cómo conforman el espacio acústico utilizado por los seres humanos y la vida marina (figura 1). Sin embargo, también es posible estimar un paisaje sonoro combinando las firmas acústicas de fuentes regionales. El modelado de paisajes sonoros es el proceso de componer los sonidos en un entorno basado en fuentes, ubicaciones, movimientos y condiciones de propagación acústica supuestos. Los investigadores modelarán paisajes sonoros para probar algoritmos de detección, clasificación y localización en condiciones controladas o para predecir los efectos potenciales de las actividades humanas. Tradicionalmente se han hecho supuestos de simplificación para reducir la carga computacional, especialmente para el ruido de 
la superficie del mar causado por el viento y las olas y la pérdida de propagación acústica. A medida que aumenta la velocidad de las computadoras, las fuentes de sonido más avanzadas y los modelos de propagación permiten el desarrollo cada vez más rápido de algoritmos y una mejor comprensión de la propagación del sonido, así como la autorización solicitudes de estudio de exposición incidental a la vida marina durante actividades industriales, científicas o militares (por ejemplo, figura 5). ${ }^{32}$

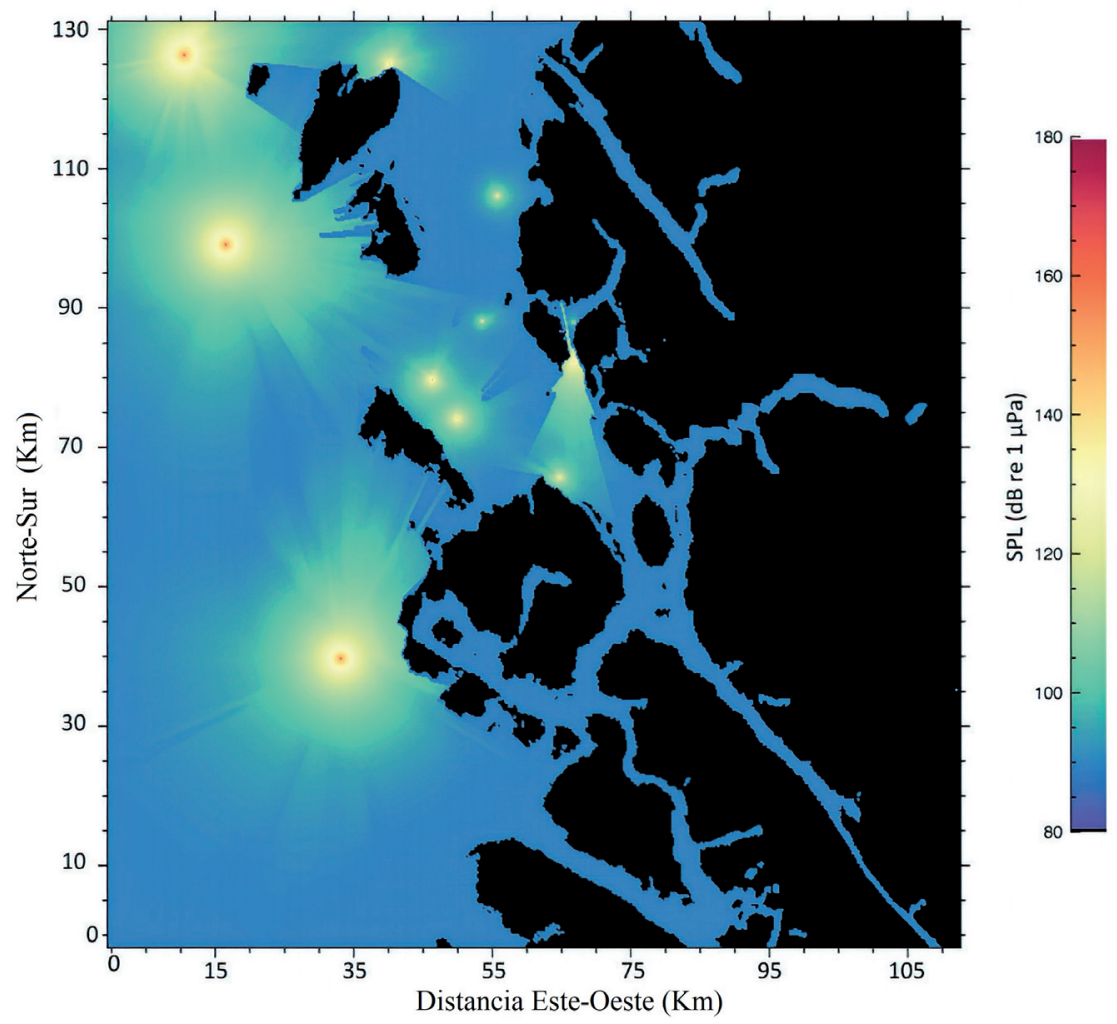

Fig. 5. Modelo del SPL a partir de una instantánea de la ubicación de los buques del sistema de identificación automatizada, que se generó como parte de la investigación de los efectos acumulativos del tráfico de buques actual y adicional proyectado en el puerto de Prince Rupert, Columbia Británica. Figura proporcionada por JASCO Applied Sciences, Nueva Escocia, Canadá, y la Autoridad Portuaria Prince Rupert.

\section{MEDICIONES ACÚSTICAS PARA LA CONSERVACIÓN}

Entender los efectos del ruido en la vida marina motiva muchas mediciones de paisajes marinos sonoros. Los efectos del ruido a menudo se agrupan en cuatro categorías: (1) muerte y lesión, (2) efectos fisiológicos, (3) alteración del comportamiento, y (4) enmascaramiento de sonidos. La protección de la vida marina contra la muerte y las lesiones ha sido el centro de atención de la industria y los fondos gubernamentales recientes. Como resultado, sabemos más sobre qué niveles de sonidos y métricas predicen lesiones, especialmente a los mamíferos marinos, que aquellos asociados con cambios de comportamiento y enmascaramiento. Se utilizan dos mediciones de umbral para estimar el conjunto de lesiones en la vida marina. El nivel máximo de presión acústica del impulso (por ejemplo, explosión o pilotaje) se utiliza para evaluar la posibilidad 
de daño fisiológico a los tejidos (por ejemplo, barotrauma en los peces). La energía sonora que puede dañar la audición en la vida marina es estimada por el SEL, que acumula energía sonora a lo largo del tiempo. ${ }^{33,34}$. El SEL en el medio marino es una dinámica complicada relacionada con la distancia de origen, las condiciones de propagación acústica y el solapamiento entre el contenido de frecuencia de la fuente y la sensibilidad auditiva de los animales receptores. Para la exposición al sonido humano, utilizamos la ponderación familiar A, y se proponen funciones de ponderación similares para cinco grupos de mamíferos marinos. ${ }^{35}$ Aún no hemos entendido lo suficiente la audición de peces, tortugas marinas e invertebrados como para proponer funciones de ponderación para estos grupos.

Los estudios de alteración del comportamiento y enmascaramiento auditivo están aumentando ahora que los efectos agudos del ruido se entienden mejor. Estos estudios están directamente asociados con el concepto de un paisaje sonoro; ¿cómo interpreta y reacciona la vida marina al sonido? La mayoría de los estudios de alteración del comportamiento relacionan la reacción con el nivel de presión sonora en el momento de la reacción. Se requiere mucho trabajo adicional para entender mejor qué medidas, incluyendo el movimiento de partículas, son apropiadas para entender la interrupción del comportamiento en la mayoría de los taxones. El enmascaramiento ocurre cuando la capacidad de detectar o reconocer un sonido de interés se degrada por la presencia de otro sonido, el enmascarador. ${ }^{36}$ Las señales submarinas pueden ser enmascaradas por componentes naturales del paisaje sonoro, como el hielo marino, las interacciones entre el viento y las olas, la lluvia, los coros de animales distantes o los ataques vocales, así como por actividades trompogénicas. Mientras que los estudios de lesiones inducidas por el sonido o trastornos del comportamiento se centran únicamente en la señal de interés, los estudios de enmascaramiento deben cuantificar el ruido ambiente para estimar la relación señal-ruido, que es crítica para el apareamiento cuando un animal puede detectar una señal.

\section{ESTANDARIZANDO LA MEDICIÓN DE LOS PAISAJES SONOROS MARINOS}

La aplicación de mediciones de paisajes sonoros en estudios que evalúan los efectos del sonido humano, cartografían la distribución de la vida marina sonífera y comprenden el papel del sonido en la ecología de la vida marina Se trata de una comunidad mucho más grande que se dedica a la monitorización acústica pasiva y al análisis del paisaje sonoro. Como resultado, la comunidad ha identificado la necesidad de desarrollar terminología y métodos estándar que ayuden a asegurar que la investigación y las mediciones de cumplimiento sean repetibles y comparables entre los proyectos. La norma 18405 de la Organización Internacional de Normalización (ISO) sobre acústica subacuática (ISO, 2017) incluye por primera vez una definición de paisaje sonoro subacuático. ${ }^{37}$

Acústica subacuática: Caracterización del sonido ambiental en términos de sus atributos espaciales, temporales y de frecuencia y los tipos de fuentes que contribuyen al campo sonoro.

El sonido ambiente es el campo sonoro medido en ausencia del ruido relacionado con el sistema de medición. El concepto de paisaje sonoro tiene 
sus raíces en la comprensión de cómo los seres humanos interpretan el entorno sonoro urbano. La definición de la acústica subacuática no incluye elementos de percepción porque no podemos saber de manera concluyente cómo la vida marina interpreta los sonidos. La norma ISO 18405 proporciona definiciones precisas de terminología acústica subacuática que ayudarán a los grupos a intercambiar resultados utilizando una notación común. ${ }^{38}$

La terminología de la norma ISO 18405 no define cómo describir un paisaje sonoro. Dada la amplia gama de métricas e índices que pueden utilizarse para describir un paisaje sonoro y la incertidumbre que rodea los efectos y la percepción del ruido, llegar a un consenso entre los investigadores llevará tiempo. El equipo de la Red Atlántica de Observación de Ecosistemas de Aguas Profundas (ADEON; https://adeon.unh.edu/) elaboró tres normas del proyecto, basadas en la terminología de la norma ISO 18405, que definen las métricas de referencia, la recopilación de datos y los datos métodos de procesamiento que el proyecto utilizará para medir y documentar el paisaje sonoro. El equipo del proyecto espera que los documentos (disponibles en el sitio web del proyecto) iniciarán un debate que conduzca a un consenso sobre la descripción mínima de un paisaje sonoro submarino, probablemente a través de un nuevo grupo de trabajo en el Comité Técnico 43 de la ISO. (actualizado en 2018)

\section{Sonido oceánico: \\ Una variable oceánica esencial}

La ampliación de los esfuerzos para medir los paisajes marinos concuerda con la mayor atención que se presta a los sistemas de observación de los océanos. El Sistema Mundial de Observación de los Océanos (SMOO) fue desarrollado por la Comisión Oceanográfica Intergubernamental de la UNESCO en torno a tres temas fundamentales para comprender mejor el clima oceánico, los ecosistemas y las repercusiones y vulnerabilidades humanas: 1) servicios en tiempo real (www.goosocean.org). El sistema coordinado de observatorios oceánicos a largo plazo se basa en un marco diseñado para ser flexible, adaptarse a la innovación científica, abordar las necesidades de la sociedad y ofrecer un sistema de observación con una base de usuarios máxima y un impacto social. El marco del SMOO relaciona todas las observaciones de los océanos con vehículos todo terreno para garantizar mediciones que atraviesan las plataformas de observación y representan el plan más rentable para proporcionar una cobertura mundial opcional para cada vehículo todo terreno. Los grupos de expertos del SMOO consideran las EOV en términos de preparación científica, relevancia social y viabilidad.

Las diversas aplicaciones de la información obtenida del estudio de los océanos inspiraron al Programa Internacional de Experimentos Oceánicos Silenciosos (www.IQOE.org) para proponer Ocean Sound como EOV al Grupo de Biología y Ecosistemas del SMOO para su inclusión en la red del SMOO. Aunque el sonido de los océanos es una medida física característica del medio marino, se consideró que el Grupo de Biología y Ecosistemas era el más apropiado para la presentación, ya que la mayoría de los productos oceánicos sanos derivados de su medición tienen aplicaciones biológicas y ecosistémicas directas o indirectas relacionadas con la economía, la alimentación, la conservación y el clima, 
y la sostenibilidad (tabla 1). Ocean Sound se ocupa de 7 de las 10 presiones sociales del SMOO y de los 9 motores sociales del SMOO (tabla 1). La escala de observación de las redes de sonido oceánico y las plataformas de grabación permitirá estudiar fenómenos que van desde eventos acústicos únicos hasta tendencias a largo plazo en el sonido ambiental (figura 6). El Ocean Sound EOV forjará importantes avances en nuestra comprensión de los paisajes sonoros oceánicos, los efectos que tiene en la vida marina y cómo se puede utilizar el monitoreo acústico para evaluar la biodiversidad y la salud de los ecosistemas.
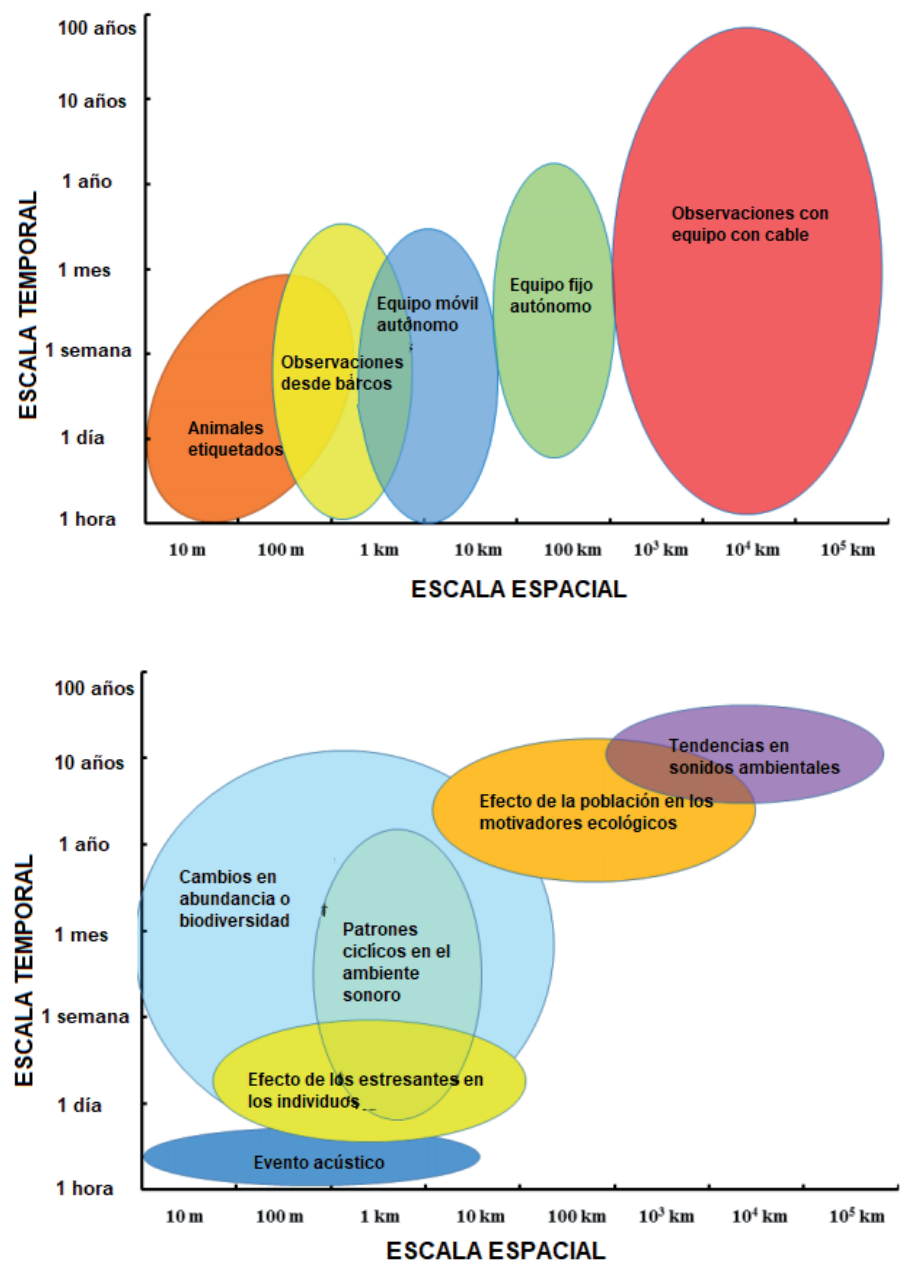

Fig. 6. Arriba: escalas observacionales de plataformas de registro de la variable oceánica esencial de Ocean Sounds (EOV). Los sensores de registro pasivo varían en tamaño y capacidad de registro desde pequeñas etiquetas de despliegue cortas conectadas directamente a los animales hasta sensores autónomos de capacidad intermedia a observatorios de gran escala con sensores cableados directamente a la costacapacidad de registro de términos. Bot-tom: fenómenos acústicos que serán captados por la gama Ocean Sound EOV en escala desde la detección acústica única de un buque que pasa o terremotos no-acuáticos hasta tendencias a largo plazo en el sonido ambiental del océano durante décadas. Ocean Sound apoya la derivación de métricas e índices acústicos que estiman la biodiversidad del ecosistema, la abundancia de ballenas cantantes y los efectos del cambio ambiental a nivel individual y poblacional. 
Tabla 1. Información básica sobre las variables oceánicas para el sonido oceánico.

\begin{tabular}{|l|l|}
\hline Subvariables & Presión acústica y movimiento de partículas \\
\hline Productos derivados & $\begin{array}{l}\text { Campo sonoro y tendencias, Niveles de presión sonora, } \\
\text { Niveles de espectro, Niveles de banda (por ejemplo, banda } \\
\text { de octava), Paisaje sonoro, Niveles de fuente, e Indicadores } \\
\text { de biodiversidad }\end{array}$ \\
\hline Variables de apoyo & $\begin{array}{l}\text { Fuentes: Distribución y características de las fuentes } \\
\text { antropógenas, abióticas y bióticas. } \\
\text { Parámetros de propagación: Perfiles de velocidad acústica; } \\
\text { Corrientes oceánicas y otros fenómenos oceanográficos } \\
\text { físicos; Condiciones frontera (por ejemplo, rugosidad de } \\
\text { la superficie del mar, características del hielo marino [por } \\
\text { ejemplo, rugosidad y espesor], y del fondo marino [batimetría, } \\
\text { propiedades geoacústicas]). } \\
\text { Receptores: Sensibilidad hidrófona en función de la frecuencia } \\
\text { y direccionalidad del sistema receptor }\end{array}$ \\
\hline Impulsores sociales & $\begin{array}{l}\text { (1) Necesidad de acceso a los conocimientos científicos y a los } \\
\text { datos, (2) Crecimiento y desarrollo económico sostenible, (3) } \\
\text { Conservación de la biodiversidad y los ecosistemas, (4) Uso } \\
\text { sostenible de la biodiversidad y los recursos en general, (5) } \\
\text { Calidad y salud medioambientales, (6) Creación de capacidad } \\
\text { y transferencia de tecnología, (7) Seguridad alimentaria, (8) } \\
\text { Prevención de amenazas y mitigación de los efectos, y (9) } \\
\text { Mejorar la gestión mediante un enfoque integrado de los } \\
\text { ecosistemas. }\end{array}$ \\
\hline Presiones sociales & $\begin{array}{l}\text { (1) Cambio climático, 2) acidificación de los océanos, 3) } \\
\text { fenómenos meteorológicos extremos, 4) pérdida de recursos } \\
\text { (hábitats y biodiversidad), 6) minería, 9) ruido y 10) desarrollo } \\
\text { costero. }\end{array}$ \\
\hline
\end{tabular}

\section{VISIÓN AL FUTURO}

En la última década se ha avanzado considerablemente en el estudio y la aplicación de los paisajes sonoros submarinos, pero todavía hay una brecha significativa en la aplicación de la construcción perceptiva de paisajes sonoros submarinos a la vida marina en términos de enmascaramiento y sentido del espacio comunitario como se refleja en la literatura del paisaje sonoro humano. El reto de integrar la percepción en las aplicaciones del paisaje sonoro subacuático se refiere a la de los colegas del paisaje sonoro terrestre que están lidiando con la percepción del paisaje sonoro en la vida silvestre. Probablemente nunca entenderemos la percepción de todos los taxones animales para identificar y cuantificar su experiencia de los paisajes submarinos y terrestres. Un paso adelante que se puede lograr es comprender mejor las capacidades auditivas y la variabilidad entre los individuos y las especies y en términos del contexto relacionado con la edad, el género, la exposición previa al ruido y el estado de comportamiento. Se trata de un esfuerzo elevado porque existen diversos órganos de protección del sonido empleados bajo el agua, por ejemplo, orejas de mamíferos similares a las nuestras, órganos otolitos en peces, y órganos estatocistas en invertebrados. Este conocimiento es fundamental para ponderar adecuadamente los paisajes sonoros de diferentes grupos de animales a fin de evaluar los efectos relacionados con la exposición al sonido o el entorno acústico cambiante. 
También es importante dejar claro que este artículo no aborda directamente el componente de movimiento de partículas del sonido en el paisaje sonoro. Sin embargo, reconocemos que este es un componente increíblemente importante del paisaje sonoro para una mayoría de taxones marinos (por ejemplo, peces, invertebrados) que sienten este componente del sonido. ${ }^{4}$ Esta brecha existe porque históricamente la instrumentación para medir el movimiento de partículas en el océano abierto no ha sido fácilmente accesible para la comunidad investigadora. A medida que la nueva tecnología esté disponible para medir este parámetro del campo sonoro bajo el agua, esperamos emocionantes avances en el conocimiento y las aplicaciones del paisaje sonoro submarino.

El último y posiblemente el desafío abierto más importante asociado con los paisajes sonoros oceánicos es cómo comprender mejor las mediciones acústicas y los modelos en seis dimensiones. La naturaleza interdisciplinaria de la investigación del paisaje sonoro debe ampliarse de nuevo para abarcar a los informáticos, a los psicoanalistas cognitivos y a los expertos en tecnología de Internet para avanzar en la percepción de paisajes sonoros submarinos o imágenes de paisajes sonoros bidimensionales para abarcar combinaciones innovadoras de representación visual y auditiva para capturar completamente la complejidad del paisaje sonoro de una manera que podamos percibir mejor.

\section{AGRADECIMIENTOS}

Agradecemos a Arthur Popper sus invaluables comentarios y comentarios sobre los borradores de este documento. Jennifer L. Miksis-Olds fue financiada por el Bureau of Ocean Energy Management Contract ADEON M16PC00003.

\section{REFERENCIAS}

1. Jennings, P., and Cain, R. (2013). A framework for improving urban soundscapes. Applied Acoustics 74(2), 293-299.

2. De la Estrategia de Ruido Oceánico de la NOAA. Disponible en http:// acousticstoday.org/nefsc

3. van Opzeeland, I. C., and Miksis-Olds, J. L. (2012). Acoustic ecology of pinnipeds in polar habitats. In Eder, D. L. (Ed.), Aquatic Animals: Biology, Habitats, and Threats. Nova Science Publishers, Inc., New York, pp. 1-52.

4. Hawkins, A. D., and Popper, A. N. (2017). A sound approach to assessing the impact of underwater noise on marine fishes and invertebrates. ICES Journal of Marine Science 74, 635-651.

5. Mann, D. A. (2012). Remote sensing of fish using passive acoustic monitoring. Acoustics Today 8(3), 8-15.

6. Au, W. W. L., and Lammers, M. O. (Eds). (2016). Listening in the Ocean. Springer-Verlag, New York.

7. Miksis-Olds, J. L., Stabeno, P. J., Napp, J. M., Pinchuk, A. I., Nystuen, J. A., Warren, J. D., and Denes, S. L. (2013a). Ecosystem response to a temporary sea ice retreat in the Bering Sea. Progress in Oceanography 111, 38-51. 
8. Staaterman, E., Paris, C. B., DeFerrari, H. A., Mann, D. A., Rice, A. N., and D’Alessandro, E. K. (2014). Celestial patterns in marine soundscapes. Marine Ecology Progress Series 508, 17-32.

9. McWilliam, J. N., and Hawkins, A. D. (2013). A comparison of inshore marine soundscapes. Journal of Experimental Marine Biology and Ecology 446, 166-176.

10.Parks, S. E., Miksis-Olds, J. L., and Denes, S. L. (2014). Assessing marine ecosystem acoustic diversity across ocean basins. Ecological Informatics 21, 81-88.

11.Harris, S. A., Shears, N. T., and Radford, C. A. (2016). Ecoacoustic indices as proxies for biodiversity on temperate reefs. Methods in Ecology and Evolution 7, 713-724.

12. Slabbekoorn, H., and Bouton, N. (2008). Soundscape orientation: A new field in need of sound investigation. Animal Behaviour 76, e5-e8.

13.Pijanowski, B. C., Villanueva-Rivera, L. J., Dumyahn, S. L., Farina, A., Krause, B. L., Napoletano, B. M., Gage, S. H., and Pieretti, N. (2011). Soundscape ecology: The science of sound in the landscape. BioScience 61, 203-216.

14. Soundscape Ecology of the Anthropocene - Hans Slabbekoorn

15.Able, K. P. (1980). Mechanisms of orientation, navigation, and homing. In Gauthreaux, S. A., Jr. (Ed.), Animal Migration, Orientation, and Navigation. Academic Press, New York, pp. 283-373.

16.Kenney, R. D., Mayo, C. A., and Winn, H. E. (2001). Migration and foraging strategies at varying spatial scales in western North Atlantic right whales: A review of hypothesis. Journal of Cetacean Research Management 2, 251260 .

17.Miksis-Olds, J. L., and Madden, L. E. (2014). Environmental predictors of ice seal presence in the Bering Sea. PLoS ONE 9, e106998.

18. Stanley, J. A., Radford, C. A., and Jeffs, A. G. (2011). Behavioural response thresholds in New Zealand crab megalopae to ambient underwater sound. PLoS ONE 6, e28572.

19.Simpson, S. D., Meekan, M. G., Jeffs, A., Montgomery, J. C., and McCauley, R. D. (2008). Settlement-stage coral reef fish prefer the higher-frequency invertebrate-generated audible component of reef noise. Animal Behaviour 75, 1861-1868.

20.Sueur, J., Pavoine, S., Hamerlynck, O., and Duvail, S. (2008). Rapid acoustic survey for biodiversity appraisal. PLoS ONE 3, e4065.

21.Stanley, J. A., Radford, C. A., and Jeffs, A. G. (2012). Location, location, location: Finding a suitable home among the noise. Proceedings of the Royal Society of London B: Biological Sciences 270, 3622-3631.

22.Ross, D. (2005). Ship sources of ambient noise. IEEE Journal of Ocean Engineering 30, 257-261.

23. McDonald, M. A., Hildebrand, J. A., and Wiggins, S. M. (2006). Increases in deep ocean ambient noise in the Northwest Pacific west of San Nicolas Island, California. The Journal of the Acoustical Society of America 120, 711-717. 
24.Chapman, N. R., and Price, A. (2011). Low frequency deep ocean ambient noise trend in the Northeast Pacific Ocean. The Journal of the Acoustical Society of America 129, EL161-EL165.

25.Andrew, R. K., Howe, B. M., and Mercer, J. A. (2011). Long-time trends in ship traffic noise for four sites off the North American West Coast. The Journal of the Acoustical Society of America 129, 642-651.

26. Ross, D. (1993). On ocean underwater ambient noise. Acoustic Bulletin 18, 5-8.

27.Boyd, I. L., Frisk, G., Urban, E., Tyack, P., Ausubel, J., Seeyave, S., Cato, D., Southall, B., Weise, M., Andrew, R., Akamatsu, T., Dekeling, R., Erbe, C., Farmer, D., Gentry, R., Gross, T., Hawkins, A., Li, F., Metcalf, K., Miller, J. H., Moretti, D., Rodrigo, C., and Shinke, T. (2011). An International Quiet Ocean Experiment. Oceanography 24, 174-181.

28. Tyack, P. L. (2008). Implications for marine mammals of large-scale changes in the marine acoustic environment. Journal of Mammalogy 89, 549-558.

29.Dziak, R., Fowler, M. J., Matsumoto, H., Bohnenstiehl, D. R., Park, M., Warren, K., and Lee, W. S. (2013). Life and death sounds of iceberg A53a. Oceanography 26, 10-12.

30.Miksis-Olds, J. L., Bradley, D. L., and Niu, X. M. (2013b). Decadal trends in Indian Ocean ambient sound. The Journal of the Acoustical Society of America 134, 3464-3475.

31.Miksis-Olds, J. L., and Nichols, S. M. (2016). Is low frequency ocean sound in- creasing globally? The Journal of the Acoustical Society of America 139, 501- 511.

32.Aulanier, F., Simard, Y., Roy, N., Gervaise, C., and Bandet, M. (2017). Effects of shipping on marine acoustic habitats in Canadian Arctic estimated via probabilistic modeling and mapping. Marine Pollution Bulletin 125(1-2), 115-131.

33.Popper, A. N., Hawkins, A. D., Fay, R. R., Mann, D. A., Bartol, S., Carlson, T. J., Coombs, S., Ellison, W. T., Gentry, R. L., Halvorsen, M. B., Lokkeborg, S., Rogers, P., Southall, B. L., Zeddies, D. G., and Tavolga, W. N. (2014). ASA S3/SC1.4 TR-2014 Sound Exposure Guidelines for Fishes and Sea Turtles: A Technical Report Prepared by ANSI-Accredited Standards Committee S3/SC1 and Registered with ANSI. Springer International Publishing, Cham, Switzerland.

34. National Marine Fisheries Service (NMFS). (2016). Technical Guidance for Assessing the Effects of Anthropogenic Sound on Marine Mammal Hearing: Underwater Acoustic Thresholds for Onset of Permanent and Temporary Threshold Shifts. NOAA Technical Memorandum NMFS-OPR-55, NOAA, US Department of Commerce, Silver Spring, MD. Available at http:// acousticstoday.org/noaaagt. Accessed October 16, 2017.

35. Southall, B. L., Bowles, A. E., Ellison, W. T., Finneran, J. J., Gentry, R. L., Greene, C. R., Jr., Kastak, D., Ketten, D. R., Miller, J. H., Nachtigall, P. E., and Richardson, W. J. (2007). Marine mammal noise exposure criteria: Initial scientific recommendations. Aquatic Mammals 33(4), 411-521. 
Explorando el océano a través de paisajes sonoros / Jennifer L. Miksis-Olds, et al.

36.Dooling, R. J., Leek, M. R., and Popper, A. N. (2015). Effects of noise on fishes: What we can learn from humans and birds. Integrative Zoology 10, 29-37.

37. International Organization for Standardization (ISO). (2017).

38.Underwater Acoustics-Terminology. Standard ISO/DIS 18405.2:2017, prepared by Technical Committee ISO/TC 43, Acoustics, Subcommittee SC 3, Under- water Acoustics, ISO, Geneva, Switzerland. Available at https://www. iso. org/standard/62406.html. Accessed October 16, 2017. 